\title{
Path to protection
}

\author{
The conservation community is engaged in essential debate on realistic paths to effective and equitable protection \\ of biodiversity. This must be matched with clear and workable messages to policymakers and the public.
}

T he question of how to preserve our planet's biodiversity is staggeringly complex. How much protection is needed and where? Who sets the boundaries? How will protection be monitored and enforced? What happens to human activities in protected regions? Indeed, the realization is dawning that setting targets for protected areas requires global consideration - biodiversity is not equally spread across political borders, and even full protection within large designated areas may be of little consequence if unchecked human activity, with its habitat destruction, pollution and climate change, continues elsewhere.

Discussions around protected-area policy are gathering pace as the months dwindle before the 2020 Conference of the Parties of the Convention of Biological Diversity (CBD). This meeting will update the Strategic Plan for Biodiversity 2011-2020, which set out the 20 Aichi Biodiversity Targets. Although several of the targets are related to areal safeguarding, it is Target 11 - with its aim to effectively and equitably conserve at least $10 \%$ of the global coastal and marine realm and $17 \%$ of terrestrial and inland water areas - that has become the focus of protected-area research and policy.

The simple numbers that comprise Target 11 have spurred policymakers to increase protected-area pledges: several heads of state, including Canada's Justin Trudeau and Mexico's Enrique Peña Nieto, have taken pride in their country meeting or exceeding these targets under their watch. And the statistics do show significant progress. The World Database on Protected Areas (WDPA; https://www.protectedplanet.net), managed by the United Nations

Environment World Conservation

Monitoring Centre, reveals that, at the end of 2017, marine protected areas accounted for $6.96 \%$ of the global ocean, and $15 \%$ of terrestrial regions were protected.

Yet these numbers are a broad brush. Only $1.18 \%$ of the global high seas were protected at the end of 2017 , and a single protected area in the Ross Sea accounted for $58 \%$ of this
(https://go.nature.com/2uORcWA). The WDPA is an invaluable resource, yet it can only reflect the content of submitted reports: there are inevitable lags, and the picture is dynamic as protected areas are gained and lost. Furthermore, the database presents both designated and proposed sites, and cannot delineate sites by levels of protection, which vary hugely. The equity and efficacy components of Target 11 are not reflected by numbers alone, nor are the target's aims to ensure that protected areas are ecologically representative and well-connected.

In a Comment in this issue, Barnes et al. argue that area-based prioritization risks unintended perverse outcomes. Some of these are ecological: areas of low biodiversity value may be protected rather than highly diverse or threatened regions; or biodiversity loss may continue but be undetected in a protected area. Others are political or social: expanding protected areas without expanding management capacity reduces efficacy; and continued expansion may increase apathy or resistance to protected areas. The authors propose that a shift from quantity to quality is needed to maximize the impact of protection.

Barnes and colleagues also argue that targets and indicators need to be more explicitly connected with desired conservation outcomes. In a Perspective in this issue, Driscoll et al. introduce a biodiversity-crisis hierarchy that is designed to evaluate the indicators of all 20 Aichi targets. The hierarchy presents the layers of agents that feed into biodiversity responses, from human population size and percapita resource consumption as the two fundamental drivers of biodiversity loss to ten primary threats, including agriculture, invasive species and residential and commercial development. When the authors mapped the 147 indicators for measuring progress towards the Aichi targets onto these components, they identified only consumption as being 'highly covered' by the indicators. Four components (government, corruption, energy production and mining, and transportation and service corridors) were not represented by any of the Aichi indicators. The authors also present a stepwise process by which their hierarchy can be used to identify gaps where new targets and goals are needed.

Driscoll and colleagues' use of the term 'biodiversity crisis' is no exaggeration. In March 2018, the Intergovernmental Science-Policy Platform on Biodiversity and Ecosystem Services (IPBES) presented five reports on the status of global biodiversity and ecosystem services (https://go.nature. com/2qcLYyb). The overwhelming picture is one of degradation, reduction and loss on the side of biodiversity, with continued increases in every form of human-induced pressure. The substantial uptick in designation of protected areas in the past decade will help, but it is so, so far from being enough.

Policymakers need solutions to work towards, not problems to ponder. Positive messages can inspire and empower change across the community (https://conservationoptimism.com). If these solutions are to be evidence-driven and effective, they must come from the research community. Some may argue that a modest increase from Target 11 will continue to drive protected-area expansion through being achievable. Others claim that nature needs half of the planet to be protected (https://natureneedshalf.org). And others are pushing for the focus to shift to sustainable use of the whole planet, rather than through the lens of protecting specific areas. The way forwards will be some combination of these and it may seem impossible to summarize these complex issues. But continuing research need not be at odds with an overarching message - and biodiversity needs both. Specific and simple demands are essential to communicating the urgent need for biodiversity protection and for impelling action. Between now and the 2020 CBD conference, a consensus on protection must be reached that can make headlines in every language.

Published online: 20 April 2018 https://doi.org/10.1038/s41559-018-0555-X 